\title{
Adaptive Locomotor Plasticity in Chronic Spinal Cats after Ankle Extensors Neurectomy
}

\author{
Laurent J. G. Bouyer, ${ }^{1}$ Patrick J. Whelan, ${ }^{2}$ Keir G. Pearson, ${ }^{3}$ and Serge Rossignol ${ }^{1}$ \\ ${ }^{1}$ Centre de Recherche en Sciences Neurologiques, Department of Physiology, Université de Montréal, Montreal, Quebec, \\ Canada H3C 3J7, '2Department of Physiology and Biophysics, University of Calgary, Calgary, Alberta, Canada T2N 4N1, \\ and ${ }^{3}$ Department of Physiology, University of Alberta, Edmonton, Alberta, Canada T6G 2H7
}

\begin{abstract}
After lateral gastrocnemius-soleus (LGS) nerve section in intact cats, a rapid locomotor compensation involving synergistic muscles occurs and is accompanied by spinal reflex changes. Only some of these changes are maintained after acute spinalization, indicating the involvement of descending pathways in functional recovery. Here, we address whether the development of these adaptive changes is dependent on descending pathways. The left LGS nerve was cut in three chronic spinal cats. Combined kinematics and electromyographic (EMG) recordings were obtained before and for $8 \mathrm{~d}$ after the neurectomy. An increased yield at the ankle was present early after neurectomy and, as in nonspinal cats, was gradually reduced within $8 \mathrm{~d}$. Compensation involved transient changes in step cycle structure and a longer term increase in postcontact medial gastrocnemius (MG) EMG activity. Precontact MG EMG only
\end{abstract}

In previous studies, it was demonstrated that cats (Wetzel et al., 1973) and rabbits (Stewart, 1937) could rapidly (within 2 weeks) recover relatively normal locomotor movements after an ankle extensor nerve cut. Pearson et al. (1999) determined that increased activity in a remaining agonist, medial gastrocnemius (MG), was correlated with locomotor recovery. This increase in amplitude followed a differential time course for the pre and post foot contact portions of the burst. These authors suggested that "phasic sensory feedback from the MG muscle spindles during stance acts as an error signal to rescale the magnitude of the initial, centrally generated component of the MG EMG."

In addition, compensation was associated with plastic changes in group I reflex pathways. In walking cats, stimulation of extensor group I afferents normally prolongs stance (Conway et al., 1987; Guertin et al., 1995; Whelan et al., 1995a) through the opening of a locomotor-dependent reflex pathway (Gossard et al., 1994). After sectioning of the lateral gastrocnemius-soleus (LGS) nerve, the efficacy of such stimulation is reorganized. LGS afferents, normally having the largest effects, lose their efficacy within

Received Sept. 22, 2000; revised Feb. 27, 2001; accepted March 6, 2001.

This work was supported by grants from the Canadian Institutes of Health Research (CIHR) and the Province of Quebec le Fonds pour la Formation de Chercheurs et l'Aide à la Recherche. L.J.B. was a fellow of the Canadian Neurotrauma Research Program-CIHR partnership. P.J.W. was a fellow of Natural Sciences and Engineering Research Council of Canada and Alberta Heritage Foundation for Medical Research. We thank the anonymous reviewers for valuable comments on this manuscript and Janyne Provencher, France Lebel, Jeanne Faubert, Philippe Drapeau, and Claude Gagner for technical assistance.

Correspondence should be addressed to Dr. Laurent Bouyer, Centre de Recherche en Sciences Neurologiques, Department of Physiology, Université de Montréal CP 6128, Succursale Centre-ville, Montreal, Quebec, Canada H3C 3J7. E-mail: bouyerl@magellan.umontreal.ca.

Copyright (C) 2001 Society for Neuroscience 0270-6474/01/213531-11\$15.00/0 increased in one of three cats. In a terminal experiment, the influence of group I afferents from MG and LGS on stance duration was measured in two cats. LGS effectiveness at increasing stance duration was largely decreased in both cats. MG effectiveness was only slightly changed: increased in one cat and decreased in another. In cat 3 , the plantaris nerve was cut after LGS recovery. The recovery time courses from both neurectomies were similar $(p>0.8)$, suggesting that this spinal compensation is likely a generalizable adaptive strategy. From a functional perspective, the spinal cord therefore must be considered capable of adaptive locomotor plasticity after motor nerve lesions. This finding is of prime importance to the understanding of functional plasticity after spinal injury.

Key words: locomotion; spinal cats; functional plasticity; neurectomy; reflex; EMG

$3 \mathrm{~d}$ after the neurectomy, whereas MG afferents become more efficient after $5 \mathrm{~d}$ (Whelan et al., 1995b; Whelan and Pearson, 1997). After acute spinalization, the decreased effectiveness of LGS remained, whereas the increased effectiveness of MG was only retained in two of five cats (Whelan and Pearson, 1997). These results raised questions regarding the respective roles of spinal and supraspinal structures in the initiation and maintenance of plastic changes during locomotor compensation.

As part of an effort to understand the plasticity in pathways necessary for locomotor recovery after spinal injury, the present study used chronic spinal cats (spinalized at T13) to address the issue of whether the lumbar locomotor circuits can be induced to compensate in the absence of supraspinal control.

Previous work using this model (Barbeau and Rossignol, 1987; Bélanger et al., 1996; Rossignol et al., 2000) demonstrated that the spinal locomotor circuitry is capable of a certain level of plasticity in the absence of supraspinal inputs. Indeed, Chau et al. (1998) showed that early step training can affect both the quality and rate of expression of spinal locomotion. Furthermore, this plasticity can be very specific. Hodgson et al. (1994) have shown that spinal cats could be differentially trained at stepping or standing. Yet, spinal plasticity has limits. Bouyer and Rossignol (1998) chronically removed all cutaneous inputs from the hindpaws of normal cats. The latter rapidly compensated their locomotion, but a permanent deficit in ankle flexors activation became visible when the cats were subsequently spinalized. This deficit remained, despite months of training in the spinal state.

The main objective of the present study was to examine whether chronic spinal animals could compensate after a gait deficit induced by cutting an extensor nerve. The results show that 
spinal cats recover rapidly and extensively, demonstrating that for some injuries the spinal circuitry is sufficient to detect and correct for changes in gait.

Part of these results appeared previously in abstract form (Rossignol et al., 1997).

\section{MATERIALS AND METHODS}

Animals. Three adult outbred cats of either sex (weight $3-4 \mathrm{~kg}$ ) were used in this study, for a total of 58 recording sessions. The experimental protocol was in accordance with the guidelines of the animal Ethics Committee of the University of Montreal. All surgical procedures were performed under general anesthesia (isoflurane $2 \%$ ) and aseptic conditions. After surgery, analgesic [buprenorphine hydrochloride (Temgesic), 0.005-0.01 mg/kg; Reckitt \& Colman Pharmaceuticals, Hull, UK] was given every $6-8 \mathrm{hr}$ for $1-2 \mathrm{~d}$.

Implantation. Chronic electromyographic (EMG) electrodes were implanted in selected muscles of the hindlimbs. For each muscle, a pair of Teflon-insulated multistrain fine wires (AS633; Cooner Wire, Chatsworth, CA) was led subcutaneously from head-mounted multipin connectors (Cinch Connectors; TTI Inc., Pointe-Claire, Canada) and sown into the belly of the muscle for bipolar EMG recordings. For the bifunctional muscle sartorius, the anterior part (hip flexion and knee extension) (Pratt and Loeb, 1991) was implanted.

Spinalization. At the same time as EMG electrode implantation (cats 1-2) or $50 \mathrm{~d}$ afterward (cat 3), the spinal cord was exposed at the level of the 13 th thoracic vertebra by performing a laminectomy. Care was taken to minimize damage to the articular processes. The dura was cut open and, after local lidocaine application (Xylocain, 2\%), the spinal cord was completely transected. Hemostatic material (Surgicel) was then gently inserted inside the gap, and the muscles and skin were sown back to close the opening. Additional details have been published elsewhere (Bélanger et al., 1996). Training started no earlier than $3 \mathrm{~d}$ after surgery.

Training. After spinalization, training consisted of having the experimenter hold the hindquarters of the animal over a motorized treadmill belt and gently stimulate the perineum to evoke stepping movements. Recording sessions began once the animals had reached a steady locomotor pattern with hindquarters weight support and plantar foot placement (Barbeau and Rossignol, 1987; Bélanger et al., 1996), i.e., 22 d after spinalization for cats 1-2 and $30 \mathrm{~d}$ after spinalization for cat 3 .

Neurectomy. The left popliteal fossa was exposed by carefully separating the fat pad from the adjacent tissue, keeping the top portion attached. The LGS nerve was identified, sutured, and cut $24 \mathrm{~d}$ (cats 1-2) or $32 \mathrm{~d}$ (cat 3) after spinalization. The proximal end of the nerve was then capped with flexible vinyl polysiloxane (Reprosil; Dentsply International, Milford, DE) to prevent regrowth. The fat pad was put back in place, and the opening was closed. In cat 3 , the left plantaris nerve was cut in a second surgical procedure using a similar protocol. In a previous study (Pearson et al., 1999), two animals underwent a sham operation and showed no sign of an influence of surgical trauma or anesthesia on locomotor performance when tested $6 \mathrm{hr}$ after surgery. In this study, our cats were tested on the treadmill 24-48 hr after the neurectomy. Considering these facts and the ethics of working with spinal animals, we considered that it was not necessary to include additional sham-operated animals as part of our controls.

Kinematics. For each test session, video images of the walking animals were recorded for off-line frame-by-frame kinematic analysis. Small reflective disks were placed over bony landmarks at each joint of the left hindlimb, i.e., the iliac crest, greater trochanter, lateral epicondyle, lateral malleolus, metatarso-phalangeal joint, and the tip of the fourth toe. Joint angles and foot lifts-contacts were then reconstructed from the video images using a Peak Performance motion analysis system (Englewood, CA) with a resolution of 60 fields/sec. Knee angle was mathematically corrected for skin slippage by triangulation using postmortem measurements of leg segment lengths (femur and tibia).

Calculation of muscle length. MG muscle length was calculated according to the following formula taken from Goslow et al. (1973):

$$
M G=\sqrt{a^{2}+b^{2}+c^{2}+2 a b \cos (\phi+\psi)-2 c(a \cos \phi+b \cos \psi)},
$$

where $a$ represents length from muscle origin to knee pivot, $b$ represents length from ankle pivot to muscle insertion onto calcaneus, $c$ represents length from knee pivot to ankle pivot, $\Phi$ represents angle between femur and tibia, and $\Psi$ represents angle between tibia and calcaneum.
Chronic EMG recordings. Filtered and amplified EMG signals (100 $\mathrm{Hz}-3 \mathrm{kHz}$ bandpass; gains of 500-50K) from the implanted muscles were recorded on a Vetter Digital 4000a PCM recorder. Kinematic and EMG data were synchronized using an SMPTE time code generator (Skotel Inc.). Segments of EMG data were later digitized by a microcomputer at 1000 samples/sec per channel. Burst duration, amplitude, and timing relative to foot contact were measured later using custom software. Normalized mean amplitude was defined as the area under a rectified EMG burst divided by its duration and expressed as a percentage of control. Precontact EMG activity was defined as the normalized mean amplitude occurring from burst onset to foot contact, whereas postcontact EMG activity was the normalized mean amplitude from foot contact to the end of the burst.

Statistics. Data from two control sessions were pooled to calculate the control data point presented on each graph. All statistics presented on the data graphs are the results of an ANOVA test followed by Dunnett's post hoc test for repeated testing against this control. Therefore, the asterisks on the graphs represent statistical differences to control data only. When appropriate, another post hoc test, the Student-Newman-Keuls test (SNK) (Glantz, 1992) was also performed to evaluate differences between data points rather than against control, while taking into account the statistical effects of performing these numerous comparisons.

Terminal experiment. Nine days after the LGS neurectomy (see Results), cats 1 and 2 were prepared for a terminal acute experiment in which the effectiveness to prolong stance of the group I afferents from MG and LGS was evaluated. The methods have been described fully in a previous paper (Whelan et al., 1995b) and will only be described briefly here. Under isoflurane gas anesthesia, the nerves supplying the LGS and MG muscles of both hindlimbs were cut and embedded into stimulating cuffs. A cuff was placed around each sciatic nerve to record the electrical signal evoked from stimulating either the LGS or MG nerves. The threshold of the electrical stimulus $[1 \times \mathrm{T}$ ( $\mathrm{T}$ represents threshold)] was taken as the minimum voltage necessary to produce a visually detectable potential in the sciatic nerve. After these surgical procedures, the animal was decerebrated by transecting the brainstem at a $50^{\circ}$ angle from the edge of the superior colliculus. The isoflurane gas was discontinued at this time. The animals were then placed over a moving treadmill, and walking commenced $\sim 1-3 \mathrm{hr}$ after decerebration. Walking was facilitated by stimulation of the perineum in both animals and administration of clonidine $(25-85 \mu \mathrm{g} / \mathrm{kg}$, i.v.). In one cat, naloxone $(25 \mu \mathrm{g} / \mathrm{kg}$ ) (Pearson et al., 1992) was added to stabilize the rhythm after administration of clonidine. During bouts of walking, one of the four nerves (left and right MG, left and right LGS) was stimulated at $2 \times \mathrm{T}$ randomly through the step cycle or was synchronized to the beginning of an extensor burst in left vastus lateralis (VL). After the experiment, selected data segments were rectified, bandpass filtered $(10-100 \mathrm{~Hz})$, and stored on a computer disk using the Axotape data acquisition system (Axon Instruments, Foster City, CA). Data analyses were performed using custom programs that could retrieve data from Axotape files. The cycle periods before, during, and after the stimulus were calculated only during regular sequences of rhythmic locomotor activity. Each cycle period was calculated as the time between the occurrence of successive semitendinosus (St) bursts. All detections of the flexor bursts were made by manually tagging the onsets of the bursts using custom software. A spreadsheet program was used to calculate the mean and SD for these cycle periods, and Student's $t$ tests detected significant differences. The data were normalized according to the following equation to allow for comparisons between animals and conditions.

Effectiveness $=(b-a) /(c-a) * 100$, where $b$ equals the stimulated cycle period, $a$ represents the control cycle period, and $c$ represents the time from the onset of the first flexor burst before the stimulated extensor burst to the offset of the stimulus train (see Fig. 9).

\section{RESULTS}

Our main aim was to examine whether stepping spinal animals could compensate for a deficit in gait resulting from the LGS nerve axotomy. There was a clear yield at the ankle initially, and by the end of the first week, this deficit had almost disappeared in all of the animals. The extent of the deficit and the time course of recovery were similar to that reported previously for nonspinal cats (Pearson et al., 1999). In addition, two cats where shamoperated in that previous study, clearly establishing that operative 
A. Control

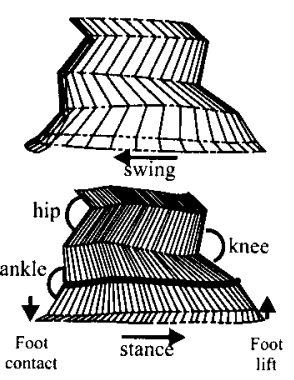

C. post neurectomy (1 day)
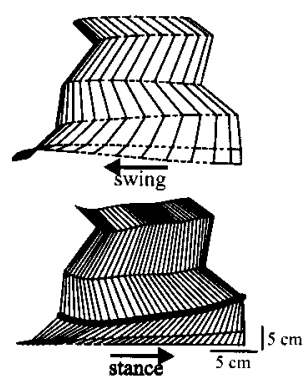

B.

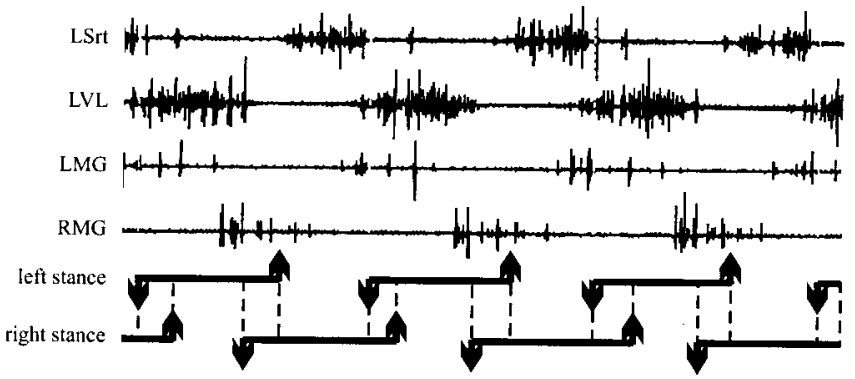

D.

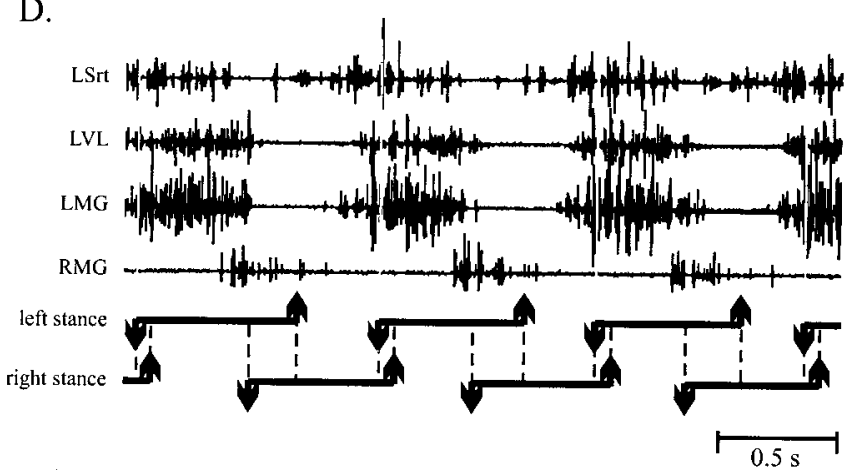

Figure 1. $A, C$. Stick figure reconstructions of the left hindlimb generated from a frame-by-frame video analysis during locomotion at a treadmill speed of 0.4 $\mathrm{m} / \mathrm{sec}$ before $(A)$ and $1 \mathrm{~d}$ after cutting the left LGS nerve $(C)$ in cat 2. $B, D$, Top traces, Raw EMG data obtained during the bouts of locomotion from which $A$ and $C$ were taken. Bottom traces, Left and right hindlimb duty cycles. Downward arrows represent foot contact, upward arrows represent foot lift, and dark horizontal lines represent stance phase. This cat was spinalized $24 \mathrm{~d}$ before the LGS neurectomy. $L M G$, Left MG; $R M G$, right $\mathrm{MG}$. trauma could not be responsible for the changes in locomotion, because these cats walked normally as early as $6 \mathrm{hr}$ after surgery.

\section{Initial deficits in gait caused by an LGS neurectomy}

Figure 1 shows an example of the kinematic and electromyographic changes that were observed early after the LGS neurectomy in the spinal cat during treadmill locomotion at $0.4 \mathrm{~m} / \mathrm{sec}$. Data shown were obtained from cat 2 in the control period and $1 \mathrm{~d}$ after cutting the LGS nerve. Stick figure diagrams reconstructed from a frame-by-frame video analysis of one complete step cycle (swing and stance phases presented separately) show the spatial relationship between the different leg segments during locomotion. The darker lines join the ankle joint marker located on the lateral malleolus from one frame to the next to emphasize ankle trajectory. In the control state, the ankle yielded very little throughout stance, as indicated by the fact that the lateral malleolus was maintained in the same vertical position. In addition, during the late portion of the stance phase, the ankle was gradually extended such that the toes reached a more extended position than the lateral malleolus at foot lift, i.e., the initiation of the swing phase (Fig. $1 A$ ).

One day after neurectomy (Fig. $1 C$ ), the ankle had a marked yield during the first half of the stance phase. In addition to the predicted yield of the ankle, there were other deficits in the gait patterns. The swing phase was initiated with the ankle joint still flexed, i.e., before the toes had passed the lateral malleolus. Figure 1, $B$ and D, shows scaled raw EMG signals from knee and ankle muscles obtained at the same time as the kinematic data shown in $A$ and $C$. In the control state, activity in the left sartorius anterior (LSrt) was mostly restricted to the swing phase. Also, the left and right MG showed small, synchronized clonic activity instead of large bursts of asynchronous activity, such as displayed by the left VL (LVL; knee extensor). This clonic behavior is often observed in spinal cats (Lovely et al., 1990; Bélanger et al., 1996). One day after the neurectomy, LSrt showed prolonged activity now covering also a large portion of the stance phase. Left MG activity was greatly augmented, whereas right MG was slightly higher, and LVL was unaffected.

The coupling between the two hindlimbs can be visualized on the duty cycle traces below the EMG data of Figure 1, $B$ and $D$. In the control state, the coupling was symmetrical, with the two hindlimbs perfectly alternating, giving rise to symmetric double support phases (areas between dashed lines in which both limbs are in contact with the treadmill and bearing weight). One day after the neurectomy, the interlimb coupling pattern was phase shifted, the right foot coming in contact with the ground earlier with respect to the left foot contact. Yet the intralimb duration of the swing and stance phases was hardly changed. This gave rise to an asymmetry in the duration of the double support phases, increasing double limb support at the transition from left to right stance (arbitrarily called double support phase 1) while simultaneously reducing the duration of double support at the right to left limb transition (double support phase 2).

\section{Kinematic compensation after neurectomy}

In Figure $2 A$, stick figure reconstructions of the stance phase illustrate the deficit and extent of recovery from day 2 to day 8 after the LGS neurectomy (cat 3). As shown for cat 2 in Figure $1 A$ (top), early after the neurectomy, the ankle yielded during early stance, and foot lift occurred before the ankle reached full extension. Yet, with daily training on the treadmill for 15-30 min, cat 3 showed a remarkably rapid recovery. The bottom stick figure shows the same cat $8 \mathrm{~d}$ after the neurectomy. The thick horizontal line joining the lateral malleolus from one frame to the next shows that the yield at the ankle had almost disappeared. Ankle extension at the end of stance was also more pronounced than at $2 \mathrm{~d}$. Figure $2 B$ shows the detailed angular excursions expressed as a function of the phase of the step cycle for the control, early ( $2 \mathrm{~d})$, and late ( $8 \mathrm{~d}$ ) conditions. Left foot contact (neurectomized leg) occurred at $\phi=0$. Note that early data in cat 3 were taken $2 \mathrm{~d}$ 

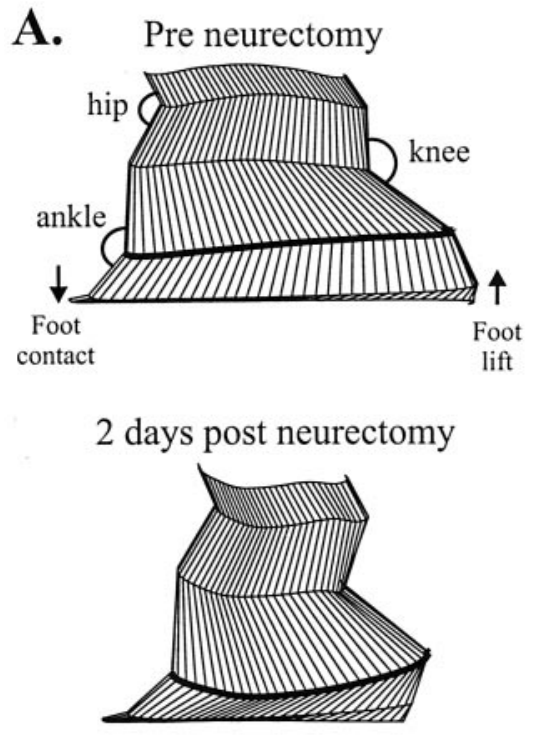

\section{8 days post neurectomy}

Figure 2. $A$, Stick figure reconstructions of the left hindlimb before (top), and $2 \mathrm{~d}$ (middle) and $8 \mathrm{~d}$ (bottom) after the neurectomy. $B$, Details of the angular excursions during the step cycle obtained before ( $n=23$; pale gray traces $)$, and $2 \mathrm{~d}(n=11$; black traces $)$ and $8 \mathrm{~d}$ ( $n=9$; dark gray traces) after the LGS neurectomy for cat 3. $C$, Time course of ankle angle $( \pm \mathrm{SD})$ at $\phi=0.3$ (which represents maximum yield) superimposed for all cats. ${ }^{*} p<0.05$.

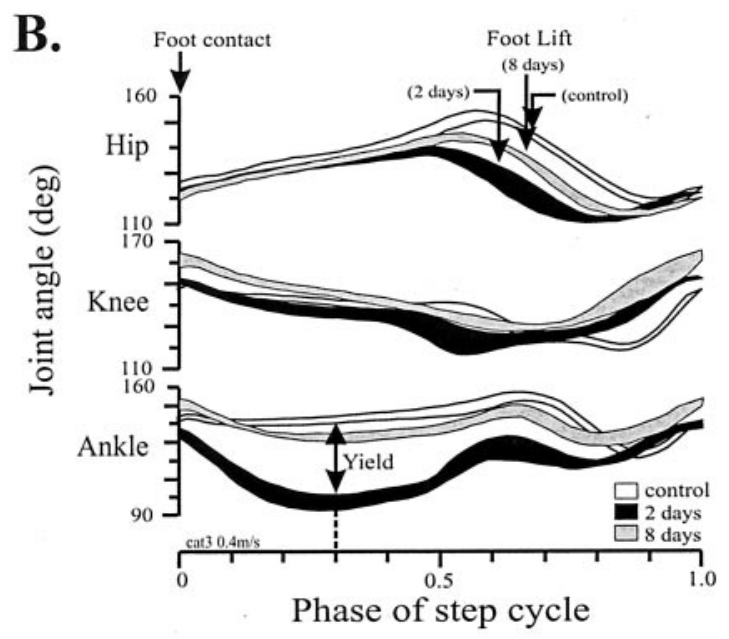

C.

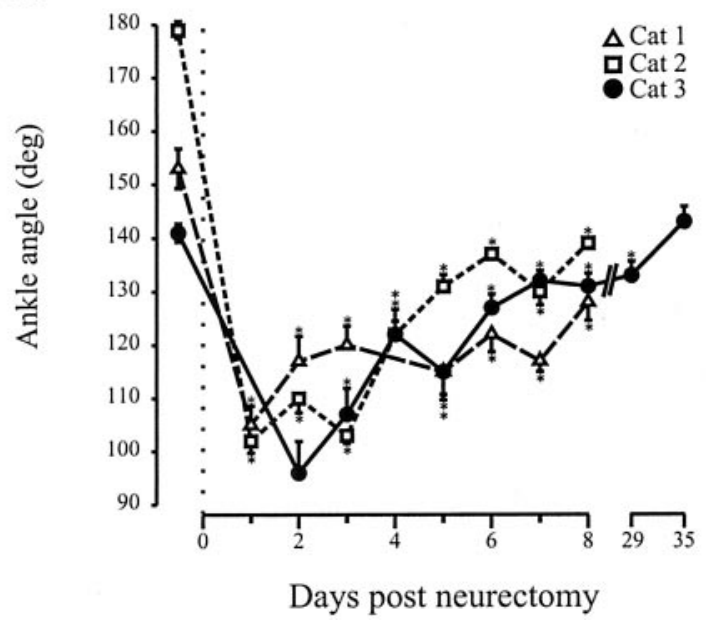

after the neurectomy because cat 3 could not walk on the treadmill on day 1. Early after the neurectomy, hip extension was reduced, and foot lift occurred prematurely. Yet, knee kinematics were hardly changed. Ankle yield started immediately on foot contact and was maximal at a phase of $\sim 0.3$ with respect to foot contact, which represents midstance. Eight days after the neurectomy, hip extension significantly improved but was still below control values. The phase of foot lift returned to its control value. The knee was slightly hyperextended during swing and early stance. At the ankle, a significant recovery occurred, but a small amount of residual yield persisted.

Figure $2 C$ shows mean ankle yield at $\phi=0.3$ as a function of time after the neurectomy in the three cats. The amount of yield caused by the neurectomy and the recovery profiles were very similar between cats. Early after the neurectomy, peak ankle position during weight bearing $(\phi=0.3)$ was close to the mechanical limit of that joint. Six to $7 \mathrm{~d}$ later, ankle angle increased to $133^{\circ}$, a value within the normal excursion range for this joint during locomotion (Goslow et al., 1973) but not identical to the control value for any of the cats $(p<0.05)$. Nevertheless, all cats had significantly improved by day 8 ( $p<0.001$; SNK test). In one animal trained for a longer period, the control value was reached $35 \mathrm{~d}$ after the LGS neurectomy $(p>0.1)$, thereby suggesting that given sufficient training time, spinal cats can completely compensate for this deficit.

The ankle joint angle was profoundly affected by the neurec- tomy, whereas the kinematics of the rest of the limb were hardly changed. For example, if one looks at the dashed line depicting $\phi=0.3$ in Figure $2 B$ and extends it to the knee and hip graphs, only small angular changes are observed at the knee, and none are observed at the hip.

\section{Gait patterns}

Although the time course of reduction in ankle yield was similar between cats, the gait patterns varied somewhat from animal to animal and sometimes from day to day after the neurectomy. Figures 3 and 4 depict these changes, looking at toe positioning and interlimb coupling, respectively. A quantification of limb segment angles during the step cycle was presented in Figure 2. Figure 3 displays toe position with respect to hip at two critical points of the step cycle, i.e., the beginning of stance (weight acceptance) and the onset of swing. Figure $3 A$ shows a schematic diagram of how the toe position was extracted from the video data by subtracting the toe marker horizontal position from the hip marker horizontal position at foot contact $(\ell 1)$ and at foot lift $(\ell 2)$. Average values for the three cats over the period of testing are plotted in Figure $3 B$. Early after the neurectomy, all cats placed the foot more rostrally with respect to the hip than during control. Figure 3, $C$ and $D$, shows the equivalent data at foot lift. Cats 2 and 3 showed a transient large reduction $(\sim 50 \%)$ in the caudal extent of the movement early after the neurectomy, which was compensated within $4-5 \mathrm{~d}$. Cat 1 showed no change in toe 


\section{A. Foot contact}

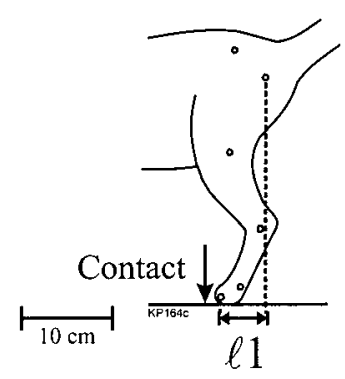

\section{Foot lift}

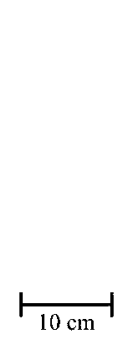

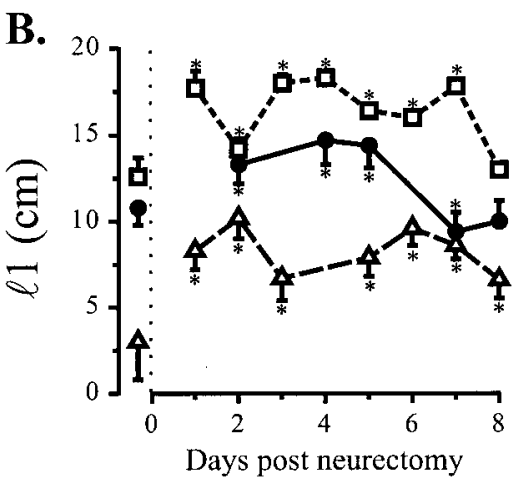

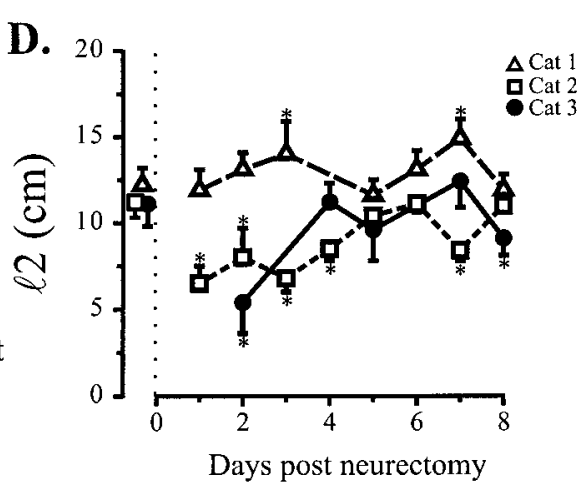

Figure 3. A, Line drawing traced from a video image taken at foot contact for cat 1 in the control situation. $B$, Average $( \pm \mathrm{SD})$ horizontal toe position with respect to the hip for the three cats. $C, D$, Same as $A$ and $B$, but for foot position at foot lift. ${ }^{*} p<0.05$. position at the onset of swing, but had the largest increase in forward toe position at landing after the neurectomy (340\% of control on day 2). So, early after the neurectomy, cats tended to place the foot of the neurectomized leg several centimeters forward and to shorten the stance phase of that limb. This shift forward in foot placement caused the cats to walk over a range of ankle angles in which the ankle extensors were more stretched, allowing a larger force output for the same level of muscle recruitment (see Discussion). These changes in biomechanics were only temporary and returned to preneurectomy values within $8 \mathrm{~d}$ in two of three cats and close to control in the other.

In Figure 1, cat 2 displayed a change in interlimb coupling $1 \mathrm{~d}$ after the lesion. Figure 4 shows in depth the interlimb coupling over time. In $A$ and $B$, the portion of the step cycle spent in double support is presented separately for the transition from left (neurectomized) leg to right (intact) leg support, arbitrarily called double support 1 (DS1; $A$ ) and from right to left (DS2;B). Clearly, only temporary changes were observed, and these were mainly an increase in DS1 for cats 2 and 3, i.e., the point at which the neurectomized leg was yielding under the weight of the hindquarters. Again, cat 1 was different, showing a reduction in both double support phases.

To understand how these changes occurred, Figure 4, $C, D$, and $E$, gives the relative phases of right foot lift, right foot contact, and left foot lift, all with respect to left foot contact. The example of asymmetric double support phases given in Figure $1 D$ resulted from an increased duration of left stance, as shown by a retarded phase of left foot lift in Figure $4 E(p<0.05)$ accompanied by an earlier onset of right stance, shown by a smaller phase value for right foot contact in Figure $4 D(p<0.05)$. Because the phase of right foot lift was also reduced (Fig. $4 C ; p<0.05$ ), the net effect was a shift of the right stance with respect to left foot contact. The general observation when looking at the three cats together is that there were variations in the pattern of alternation between the two hindlimbs in the early days after the neurectomy, but this coupling returned to normal by day 7 or 8 .

Cats sometimes changed interlimb coupling (e.g., increasing DS1 duration), thereby more rapidly unloading the neurectomized leg. However, the earliest that the right foot was actually put on the ground was at $\phi=0.42$, i.e., past the point where the peak ankle yield was measured. Therefore, the reduction in ankle yield reported in Figure 2 at $\phi=0.3$ was a true compensation by the neurectomized limb and not a change in interlimb coupling to transfer weight more rapidly onto the normal (stronger) limb.

\section{Muscle activity changes during compensation}

In Figure 5, the normalized mean amplitude of EMG activity (see Materials and Methods) of the left and right MGs and left VL and their respective burst durations are plotted against time after the neurectomy, separately for each animal. The increased activity in LSrt was transient and variable from day to day and therefore will not be presented here.

In all cats, left MG activity was greatly increased (246-621\%) immediately after the neurectomy. This increased activity remained high for cat 2 until it was killed $8 \mathrm{~d}$ later, whereas for cats 1 and 3 it came back down to a more moderate level, yet still significantly higher than control $(p<0.05)$. When animals were compared, cat 2 showed the largest increase in EMG amplitude. From Figure 3, it can be noted that this cat also placed the foot farther in front of the hip than the other two cats at the onset of stance. Similarly, cat 1, which placed the foot closest to the hip, displayed the most modest, yet still significant $(p<0.05)$, change in EMG. Cat 3 showed in-between responses both for foot kinematics and EMG amplitude. Left MG burst duration showed no concomitant change with amplitude, transiently increasing in cat 3 , decreasing in cat 2 , and remaining essentially unchanged in cat 1 .

Right MG amplitude was also increased, especially early after 

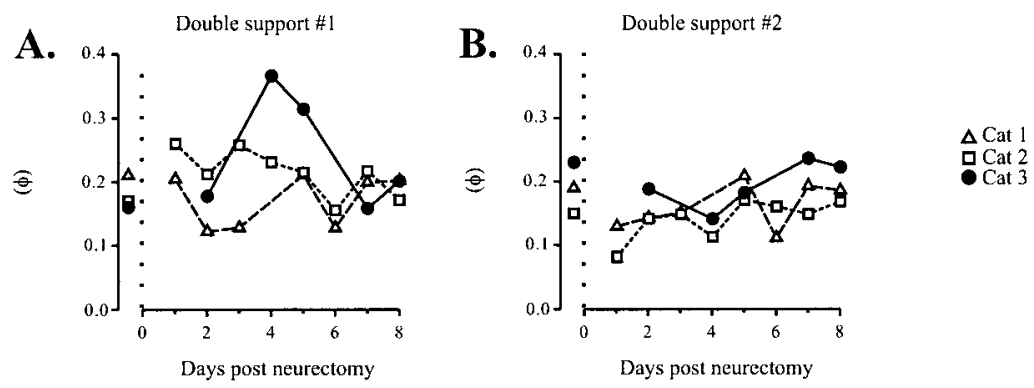
at which the two feet are on the ground) at the left to right stance transition (double support $1 ; A$ ) and right to left stance transition (double support 2; $B$ ) as a function of time after the neurectomy. $C, D, E$, Mean $( \pm \mathrm{SD})$ timing of right foot lift $(C)$, right foot contact $(D)$, and left foot lift $(E)$ with respect to left foot contact as a function of time after the neurectomy. ${ }^{*} p<0.05$.
Figure 4. Double support phases (time

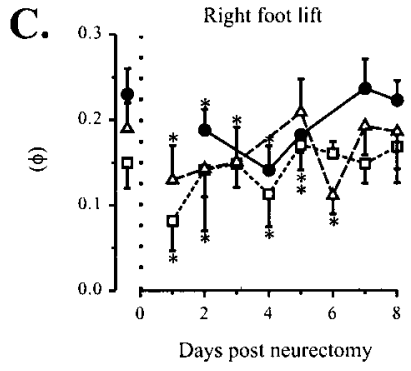

the neurectomy. However, for a given cat, this increase was always smaller than for MG on the neurectomized side. Right MG burst duration was increased in all cats early after the lesion and returned toward control values by day 5 .

Contrary to MG, the amplitude of the left knee extensor VL hardly changed after the neurectomy. Burst duration was increased in cat 2 from day 2 on, but this was associated with an increase in step cycle duration (data not shown).

\section{Precontact and postcontact MG EMG activity}

Figure 6 depicts the pre and post foot contact components of the MG EMG burst. From the rectified profiles shown on the left side of the Figure and the normalized mean amplitude differences between the control and the first point after neurectomy, it is clear that most of the changes in MG activity occurred after foot contact. The time course of changes is shown on the right of the Figure. For cats 1 and 3, the precontact component did not show a delayed increase in amplitude, but most often paralleled changes in postcontact amplitude. For cat 2, the precontact component did show a gradual augmentation over time after the neurectomy, but the major change was a large increase in precontact burst amplitude that occurred immediately after the neurectomy.

\section{Relationship between MG length and EMG amplitude}

Figure 7 shows the average length of the MG muscle at the peak of the yield ( $\phi=0.3$; see Materials and Methods) plotted along with the left MG EMG amplitude data taken from Figure 5. Early after the neurectomy, the length and EMG amplitude of $\mathrm{MG}$ were both increased. Yet, these two factors did not follow the same time course after the lesion. The vertical scale of the muscle length data is identical for all cats. Cats 1-3 showed a 50, 64, and $81 \%$ recovery, respectively. Muscle length was significantly reduced over time ( $p<0.001$; SNK test) but remained significantly larger than control even after $8 \mathrm{~d}$ for cats 1 and 2, whereas cat 3 returned to preneurectomy values.

\section{Subsequent PL neurectomy}

Although PL was not implanted, indirect evidence of an increased activation of this muscle was obtained early after the
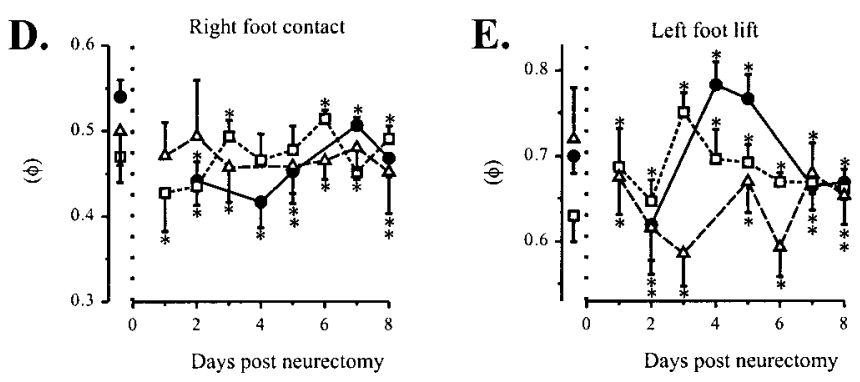

neurectomy. During the daily training periods on the first few days after the neurectomy, the cats often showed bouts of walking in which they would display an exaggerated plantar flexion of the toes at the beginning of the stance phase (data not shown). These movements would make walking irregular, and the cats would eventually fall, making these segments unsuitable for kinematic analysis.

Instead of being prepared for the acute experiment $10-11 \mathrm{~d}$ after the neurectomy, cat 3 was trained on the treadmill for an extended period of time. Thirty-six days after the LGS neurectomy, the nerve to PL was cut. In Figure $8 A$, the yields of the ankle at $\phi=0.3$ after LGS and PL neurectomies are superimposed. There is no significant difference in the time course of the two curves $(p>0.8)$. The pattern of MG EMG activity during this second compensation was qualitatively similar to what was observed after cutting LGS, with an initial large increase followed by a more modest steady increase. Quantitatively, the change in EMG activity after the PL cut was smaller than after LGS (Fig. 8, compare $B, C)$. Again, burst duration was not changed.

\section{Ankle extensor nerve stimulation}

Evidence from nonspinal animals suggests that changes in locomotor-dependent pathways occur after LGS neurectomy. These changes are evident as changes in the ability of stimulation of LGS or MG afferents to increase the duration of the stance phase (Whelan and Pearson, 1997). The purpose of this section was to test the hypothesis that similar changes in these pathways could still occur after spinalization. The effect of LGS and MG nerve stimulation on stance duration was tested in cats 1 and 2 during an acute experiment $10 \mathrm{~d}$ (cat 1) or $11 \mathrm{~d}$ (cat 2) after the neurectomy. Figure 9 presents the results obtained from these terminal experiments. In both cats, when the right (intact) LGS nerve was stimulated at two times threshold during locomotion $(A)$, cycle duration tended to be considerably increased (mean increase, 54-58\%). But when the left (neurectomized) LGS nerve was stimulated at the same intensity, cycle duration was only modestly increased (mean increase $13-16 \%, B$ and $C ; p<0.001$ ). By contrast, the results of stimulating the $\mathrm{MG}$ nerves were not 


\section{Cat 1}

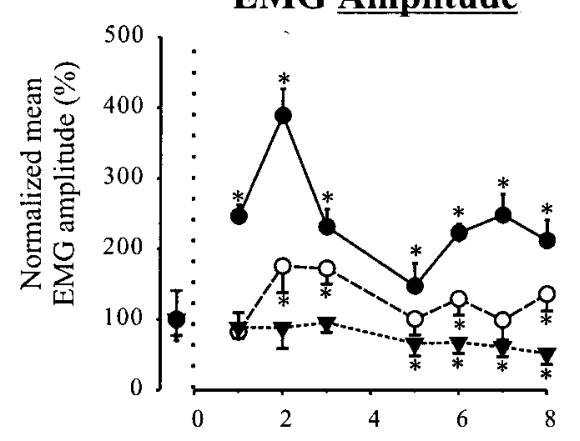

\section{EMG Duration}

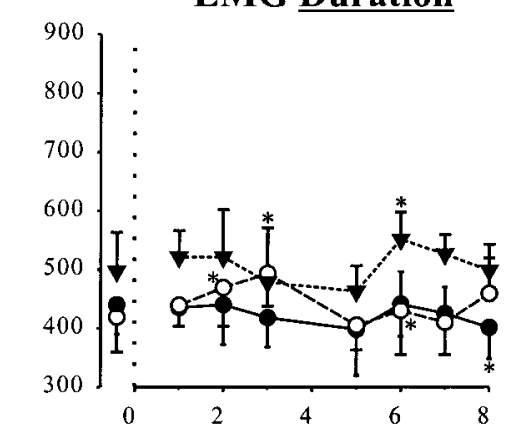

\section{Cat 2}
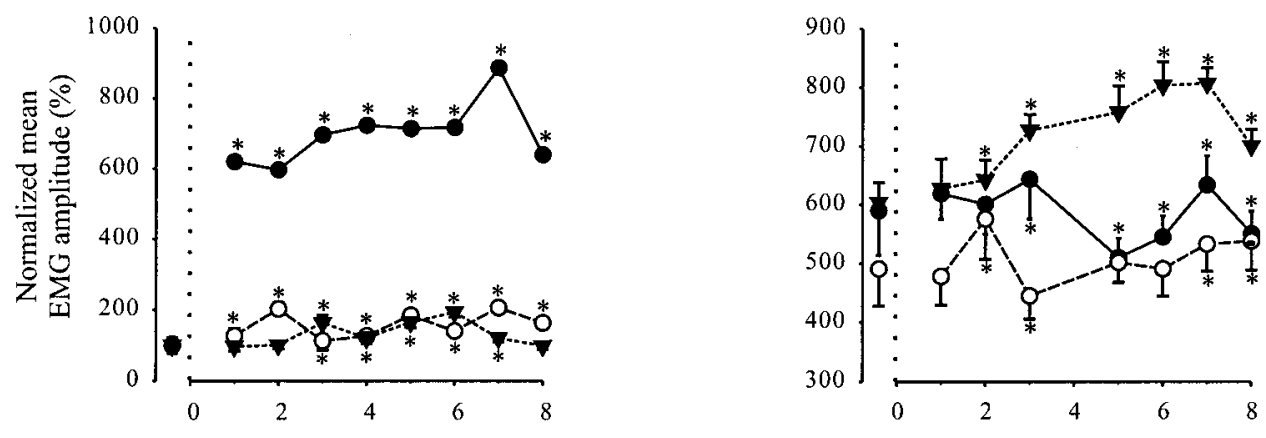

\section{Cat 3}

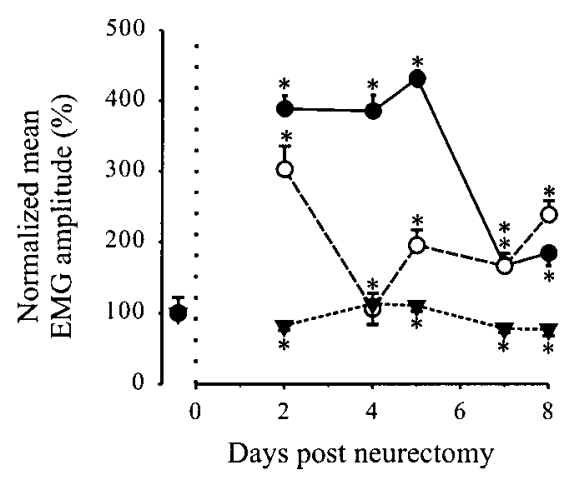

$\rightarrow-$ LMG

$-\mathrm{O}-\mathrm{RMG}$

$\rightarrow$ LVL

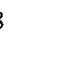




\section{Cat 1}

Figure 6. $A-C$, Average rectified EMG envelopes of the MG muscle, early (2d) and late $(8 \mathrm{~d})$ after the neurectomy in dark traces compared with control \pm SEM in gray traces. $D-F$, Time course of changes in precontact (closed circles) and postcontact (open circles) EMG as a function of time after the LGS neurectomy.

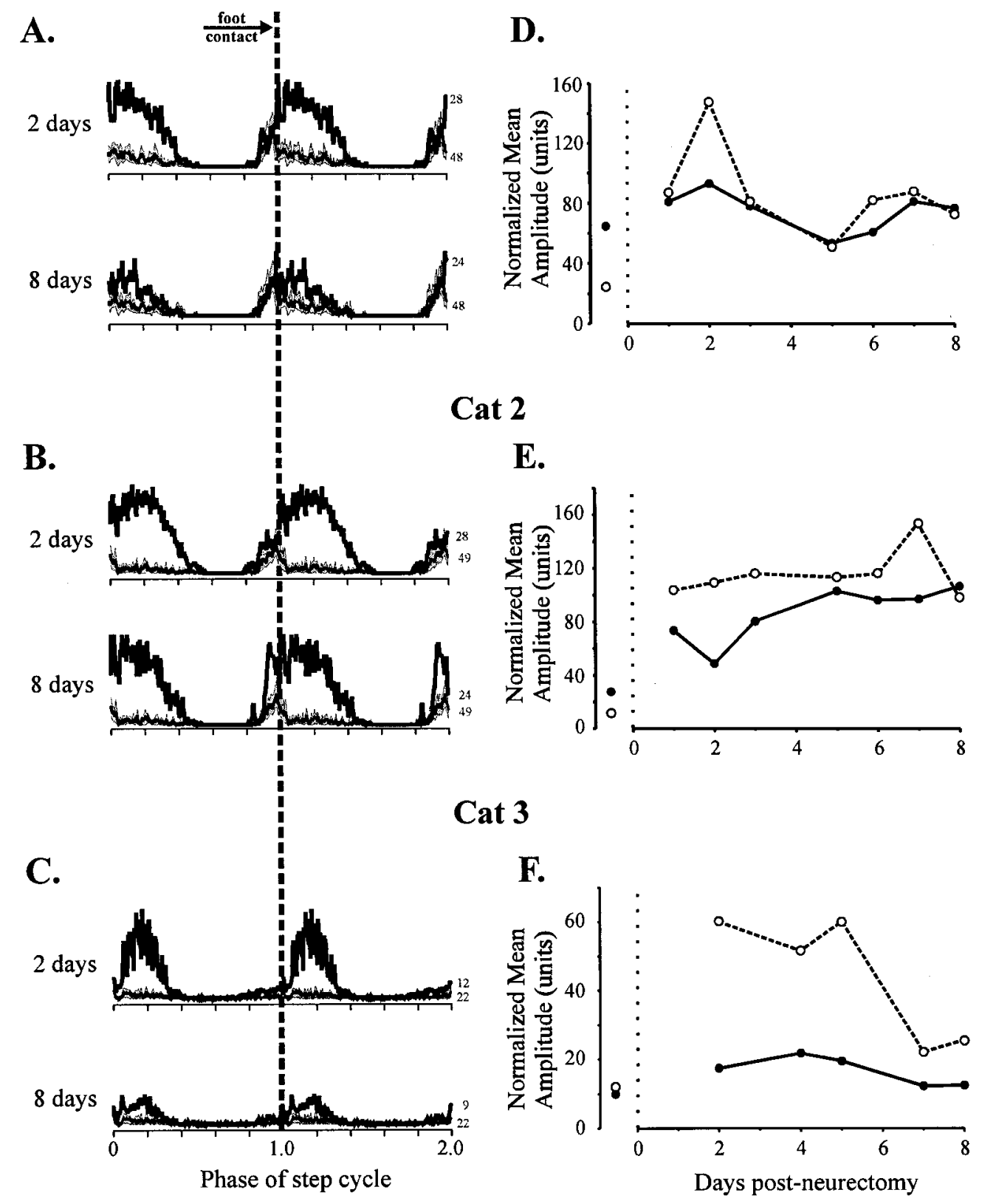

not likely to be responsible for compensation, adaptive neuronal mechanisms at the level of the spinal cord must have been actively involved.

\section{Biomechanics}

Interestingly, although the actual yield compensation after the LGS neurectomy was similar across cats, different motor strategies were used. After the neurectomy, cat 1 used shorter steps (Fig. 5) and initiated flexion of the left limb earlier in the step cycle (Fig. $4 E$ ). Cats 2 and 3 instead increased the duration of the double support period at the end of left stance (Fig. $4 A$ ).

All cats brought their left foot more rostral at the end of the swing phase (Fig. 3B). This might be related to the loss of feedback from LGS group I afferents. When stimulated during flexion in fictive locomotion, these afferents have been shown to terminate flexion and initiate extension (Guertin et al., 1995; Whelan et al., 1995a). Under normal walking conditions, these pathways could therefore participate to the control of foot landing position by sending a signal to terminate flexion to the spinal locomotor network during the lengthening contraction of the triceps surae in E1. The farther the foot is brought forward, the longer the length of the ankle extensors. Over this range of values, the longer the muscle, the larger its force-generating ability becomes. Although the origin of this lengthening might be related to a loss of sensory feedback, its seemed nevertheless to be exploited as an aid in compensation. We therefore suggest that spinal cats have access to a repertoire of motor strategies that can be used to reduce the demand for increased force generation by remaining synergists, some of them providing simple biomechanical advantages. However, their transient appearance (Figs. 3, 4) suggests that 


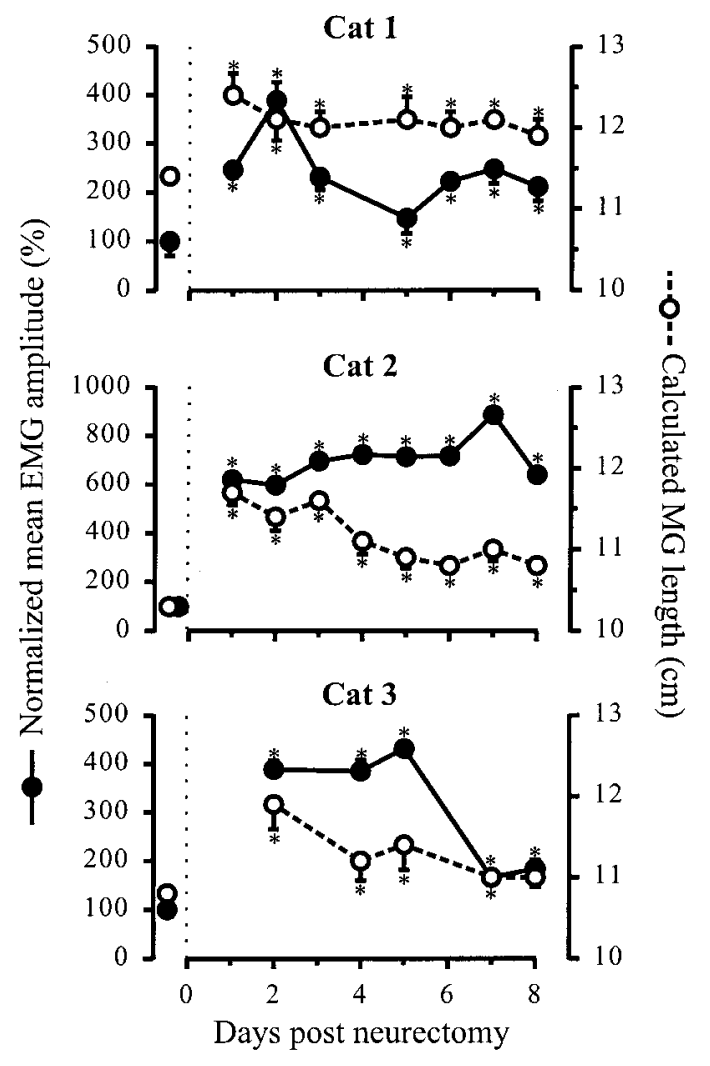

Figure 7. Normalized mean (MG EMG) amplitude $( \pm \mathrm{SD})$ and calculated MG muscle length $( \pm \mathrm{SD})$ at a normalized phase of 0.3 with respect to foot contact. ${ }^{*} p<0.05$.

they are only a temporary means of alleviating the problem until longer term neuronal adaptive mechanisms come into play.

\section{Increased muscle recruitment}

Contrary to what has been reported in nonspinal cats (Pearson et al., 1999), the increase in MG activity was not gradual over the first few days after neurectomy but peaked very early and then settled down at an intermediate level above control (Fig. 5). Also, two of the three spinal cats did not show the differential time course of increase of precontact and postcontact EMG (Fig. 6) that was noted by Pearson et al. (1999). EMG activity in spinal cats is very clonic (Fig. 1) and therefore does not allow for a detailed comparison between precontact and postcontact EMG based on individual cycles. But if averaged rectified envelopes were compared, the early component gradually increased only in cat 2. Therefore, compensation did not necessarily involve an increase of the precontact, centrally generated burst of MG EMG (Gorassini et al., 1994; Pearson et al., 1999).

Although we did not record EMG from other ankle extensors, indirect evidence suggests that they were also involved in compensation. For example, an exaggerated plantarflexion of the toes was observed during walking early after the LGS neurectomy in all cats. The distal part of the PL tendon inserts into the flexor digitorum brevis muscle, and PL is therefore considered to have a second function as a plantar flexor of the toes (Crouch, 1969). The exaggerated plantarflexion was therefore an indirect indication of increased PL activity early after the neurectomy. This increased plantar flexion disappeared over the next few days, and PL might therefore have had a late reduction in its increased activity, similarly to MG.
A.

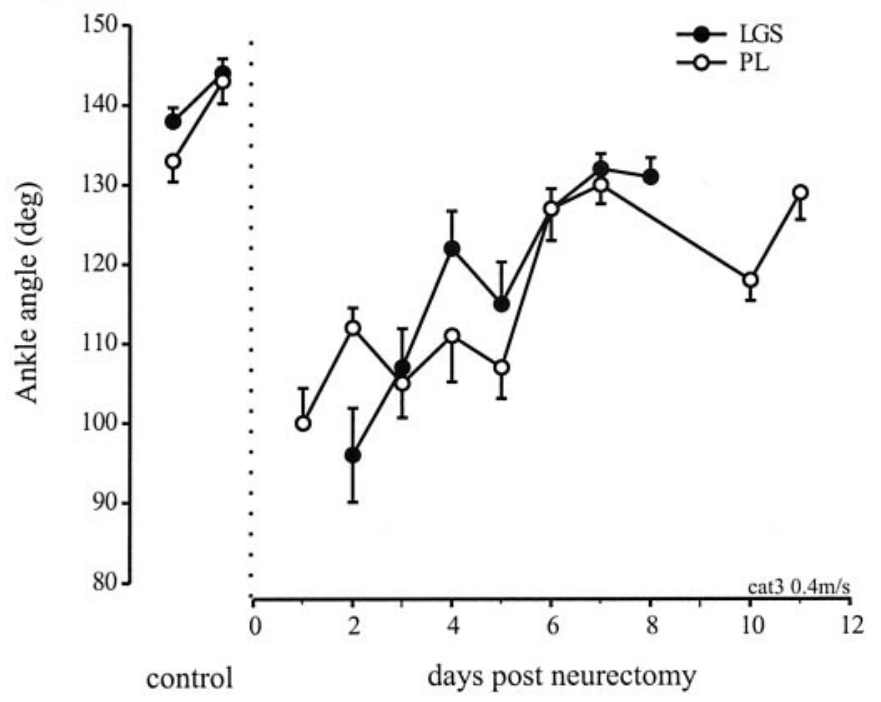

B.
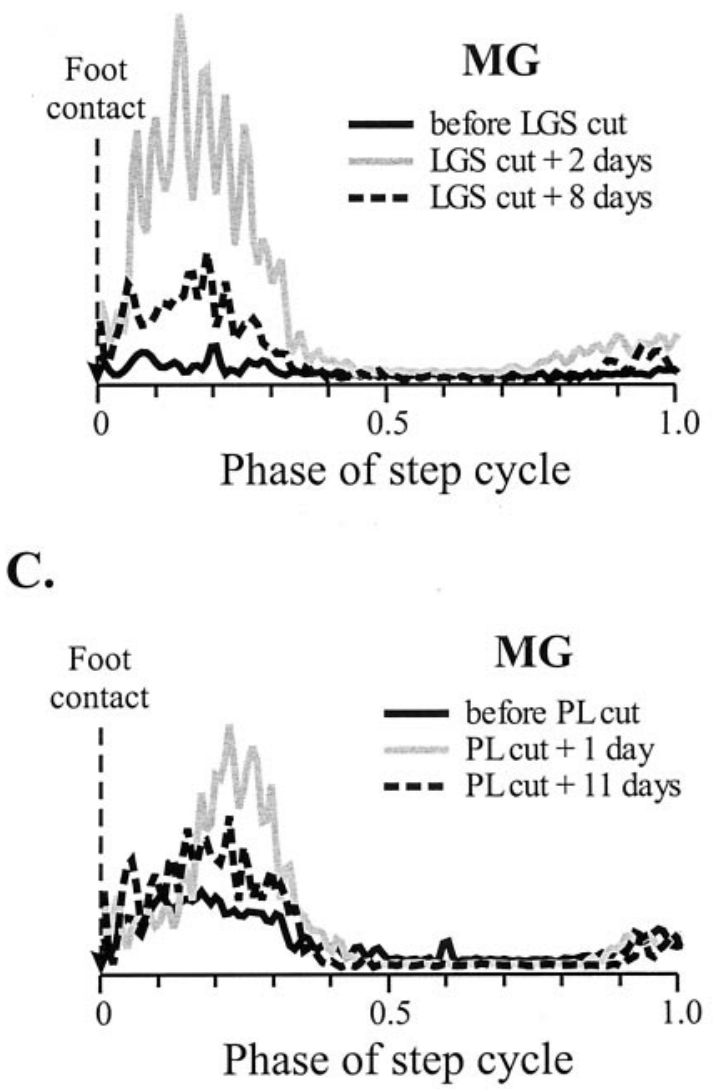

Figure 8. A, Mean ankle angles $( \pm \mathrm{SD})$ after LGS (closed circles) and subsequent PL (open circles) neurectomies in cat 3. Control, Last two walking sessions before each neurectomy.There was no statistical difference between the two curves $(p>0.8)$. $B$, Average rectified MG EMG profiles synchronized on foot contact before and 2 and $8 \mathrm{~d}$ after the LGS neurectomy. $C$, Average rectified MG EMG profiles synchronized on foot contact before and 1 and $11 \mathrm{~d}$ after the PL neurectomy. The scales are identical in $B$ and $C$. 
A. Control leg (LGS@ $2 \times$ T)

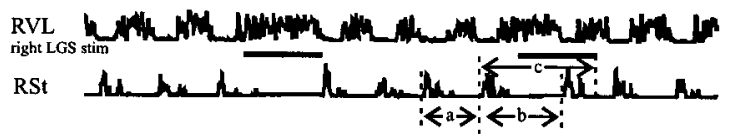

B. Neurectomized leg (LGS@ $2 \times$ T)

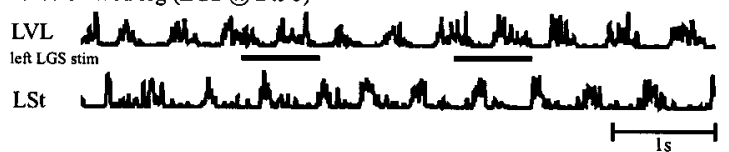

C. Effectiveness of LGS stimulation cat 1

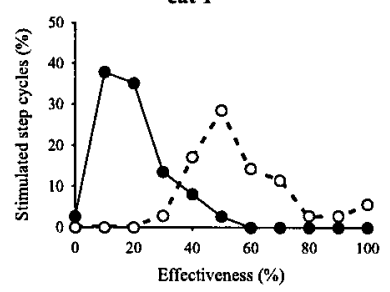

D. Effectiveness of MG stimulation

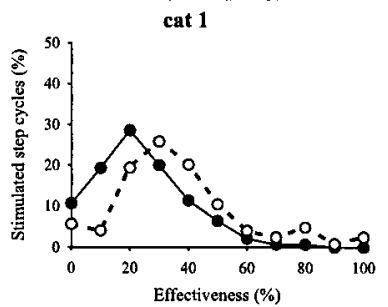

cat 2


Figure 9. A, Raw data obtained from the control (right) leg to stimulation of the right LGS nerve at $2 \times \mathrm{T}$. $B$, Raw data from the neurectomized leg obtained during stimulation of the left LGS nerve at $2 \times \mathrm{T}$. $C$, Efficiency of LGS nerve stimulation at increasing step cycle duration represented by frequency histograms. $D$, Same as $C$, but for MG stimulation. $\bigcirc$, Intact leg; $\bullet$, neurectomized leg.

\section{Increased stretch reflex feedback}

During locomotion, the stretch reflex from the triceps surae is believed to normally be responsible for $>50 \%$ of the force generated by this muscle group (Bennett et al., 1996; Hiebert and Pearson, 1999). If MG is unexpectedly stretched, such as after cutting the LGS nerve, the stretch reflex from this muscle will cause an increase in MG activity that will recruit more motor units, generate more force, and reduce ankle yield. Early after the LGS neurectomy, it is very likely that part of the increase in MG amplitude was caused by activation of the stretch reflex. Interestingly, on later days there was a clear dissociation between MG length and EMG (Fig. 7), suggesting that the change in EMG amplitude could not be attributed simply to a fixed reflex response to an increased stretch of this muscle. Therefore, although stretching of MG produced a signal to augment burst amplitude, it was not the dominating factor responsible for the long-term recovery of locomotion.

\section{Reflex pathways that could contribute to functional recovery}

Figure 8 shows that the compensations to LGS and PL neurectomies were very similar both in terms of kinematics $(A)$ and MG EMG responses $(B, C)$. The fact that $\mathrm{MG}$ behavior was similar after both lesions suggests that spinal cats might use similar compensatory mechanisms after different neurectomies. Many reflex pathways could potentially be involved in reinforcing this ongoing burst of activity in MG.

Polysynaptic group I pathways that act through the locomotor central pattern generator have been shown to change their efficacy after LGS neurectomy in nonspinal cats (Whelan et al., 1995b; Whelan and Pearson, 1997). In this study, we investigated how these were changed after a similar neurectomy in chronic spinal cats. Cats 1 and 2 used different gait strategies to achieve locomotor compensation (see above). Yet, both animals showed a similar reduction in the ability of stimulation of the neurectomized LGS nerve to increase stance duration. This result is consistent with previous reports in which decerebrate and acutely spinalized cats were used (Whelan and Pearson, 1997). Therefore, the mechanisms responsible for the reduction in LGS effectiveness must be localized in the lumbar spinal cord. The story is not so clear for the changes in MG pathways. Cat 1 showed a decrease in effectiveness, whereas cat 2 showed an increase. These changes, although statistically significant, were of small magnitude compared with what has been reported in nonspinal cats. Yet, LGS efficiency in control and neurectomized legs and MG efficiency in control leg are similar to the values reported in the literature, suggesting that the results we obtained for MG in the neurectomized leg were not attributable to experimental differences or to saturation of these pathways by the chronic spinalization.

It is important to realize that other reflex pathways that are normally involved in the regulation of locomotion but that have not yet been investigated in relation to the LGS neurectomy could also participate in compensation. They include disynaptic group I pathways from ankle extensors, which do not affect the timing of the step cycle but affect the amplitude of extensor bursts (McCrea et al., 1995; Angel et al., 1996), and cutaneous inputs from the central foot pad (Duysens and Pearson, 1976). Finally, cutaneous inputs have been found recently to become extremely important for locomotion after spinalization (Bouyer and Rossignol, 1998). Therefore, particular pathways might also be differentially recruited in the spinal and nonspinal preparations. Additional studies specifically designed to address changes in reflex transmission in these pathways have to be performed to define their role in compensation in both preparations.

\section{Conclusion}

These experiments demonstrate that chronic spinal cats are capable of adaptive locomotor plasticity after muscle nerve section, suggesting that autonomous adaptive mechanisms might be involved in long-term locomotor adaptation. This finding is of prime importance to our understanding of functional plasticity after spinal injury. Furthermore, the similitude in the kinematics between the compensated and preaxotomy states and between LGS and PL neurectomies further suggests that this spinal compensation is only one example of a generalizable adaptive strategy that tends to return the locomotor parameters to a desired kinematic pattern.

\section{REFERENCES}

Angel MJ, Guertin P, Jimenez T, McCrea DA (1996) Group I extensor afferents evoke disynaptic EPSPs in cat hindlimb extensor motorneurones during fictive locomotion. J Physiol (Lond) 494:851-861.

Armstrong RB, Marum P, Tullson P, Saubert CW (1979) Acute hypertrophic response of skeletal muscle to removal of synergists. J Appl Physiol 46:835-842.

Barbeau H, Rossignol S (1987) Recovery of locomotion after chronic spinalization in the adult cat. Brain Res 412:84-95. 
Bélanger M, Drew T, Provencher J, Rossignol S (1996) A comparison of treadmill locomotion in adult cats before and after spinal transection. J Neurophysiol 76:471-491.

Bennett DJ, De Serres SJ, Stein RB (1996) Gain of the triceps surae stretch reflex in decerebrate and spinal cats during postural and locomotor activities. J Physiol (Lond) 496:837-850.

Bouyer L, Rossignol S (1998) The contribution of cutaneous inputs to locomotion in the intact and the spinal cat. Ann NY Acad Sci 860:508-512.

Chau C, Barbeau H, Rossignol S (1998) Early locomotor training with clonidine in spinal cats. J Neurophysiol 59:392-409.

Conway BA, Hultborn H, Kiehn O (1987) Proprioceptive input resets central locomotor rhythm in the spinal cat. Exp Brain Res 68:643-656.

Crouch JE (1969) Text-atlas of cat anatomy. Philadelphia: Lea \& Febiger.

Duysens J, Pearson KG (1976) The role of cutaneous afferents from the distal hindlimb in the regulation of the step cycle of thalamic cats. Exp Brain Res 24:245-255.

Glantz SA (1992) Primer of biostatistics, Ed 3 (Jeffers JD, Englis MR, eds). New York: McGraw-Hill.

Gorassini MA, Prochazka A, Hiebert GW, Gauthier MJA (1994) Corrective responses to loss of ground support during walking. I. Intact cats. J Neurophysiol 71:603-609.

Goslow GEJ, Reinking RM, Stuart DG (1973) The cat step cycle: hind limb joint angles and muscle lengths during unrestrained locomotion. J Morphol 141:1-42.

Gossard J-P, Brownstone RM, Barajon I, Hultborn H (1994) Transmission in a locomotor-related group Ib pathway from hindlimb extensor muscles in the cat. Exp Brain Res 98:213-228.

Guertin P, Angel MJ, Perreault MC, McCrea DA (1995) Ankle extensor group I afferents excite extensors throughout the hindlimb during fictive locomotion in the cat. J Physiol (Lond) 487:197-209.

Hiebert GW, Pearson KG (1999) Contribution of sensory feedback to the generation of extensor activity during walking in the decerebrate cat. J Neurophysiol 81:758-770.

Hodgson JA, Roy RR, de Leon RD, Dobkin B, Edgerton VR (1994) Can the mammalian lumbar spinal cord learn a motor task? Med Sci Sports Exer 26:1491-1497.

Lovely RG, Gregor RJ, Roy RR, Edgerton VR (1990) Weight-bearing hindlimb stepping in treadmill-exercised adult spinal cat. Brain Res 514:206-218.

McCrea DA, Shefchyk SJ, Stephens MJ, Pearson KG (1995) Disynaptic group I excitation of synergist ankle extensor motoneurones during fictive locomotion in the cat. J Physiol (Lond) 487:527-539.

Noble EG, Tang Q, Taylor PB (1984) Protein synthesis in compensatory hypertrophy of rat plantaris. Can J Physiol Pharmacol 62:1178-1182.

Pearson KG, Jiang W, Ramirez JM (1992) The use of naloxone to facilitate the generation of the locomotor rhythm in spinal cats. J Neurosci Methods 42:75-81.

Pearson KG, Fouad K, Misiaszek JE (1999) Adaptive changes in motor activity associated with functional recovery following muscle denervation in walking cats. J Neurophysiol 82:370-381.

Pratt CA, Loeb GE (1991) Functionally complex muscles of the cat hindlimb. I. Patterns of activation across sartorius. Exp Brain Res $85: 243-256$.

Rossignol S, Bouyer L, Whelan PJ, Pearson KG (1997) Chronic spinal cats can recover locomotor function following transection of an extensor nerve. Soc Neurosci Abstr 23:761.

Rossignol S, Bélanger M, Chau C, Giroux N, Brustein E, Bouyer L, Grenier C-A, Drew T, Barbeau H, Reader T (2000) The spinal cat. In: Neurobiology of spinal cord injury (Kalb RG, Strittmatter SM, eds), pp 57-87. Totowa, NJ: Humana.

Roy RR, Baldwin KM, Edgerton VR (1991) The plasticity of skeletal muscle: effects of neuromuscular activity. Exerc Sport Sci Rev 19:269-312.

Stewart D (1937) Variations from normal gait after muscle section in rabbits. J Anat 72:101-108.

Wetzel MC, Gerlach RL, Stern LZ, Hannapel LK (1973) Behavior and histochemistry of functionally isolated cat ankle extensors. Exp Neurol 39:223-233

Whelan PJ, Pearson KG (1997) Plasticity in reflex pathways controlling stepping in the cat. J Neurophysiol 78:1643-1650.

Whelan PJ, Hiebert GW, Pearson KG (1995a) Stimulation of the group I extensor afferents prolongs the stance phase in walking cats. Exp Brain Res 103:20-30.

Whelan PJ, Hiebert GW, Pearson KG (1995b) Plasticity of the extensor group I pathway controlling the stance to swing transition in the cat. J Neurophysiol 74:2782-2787. 